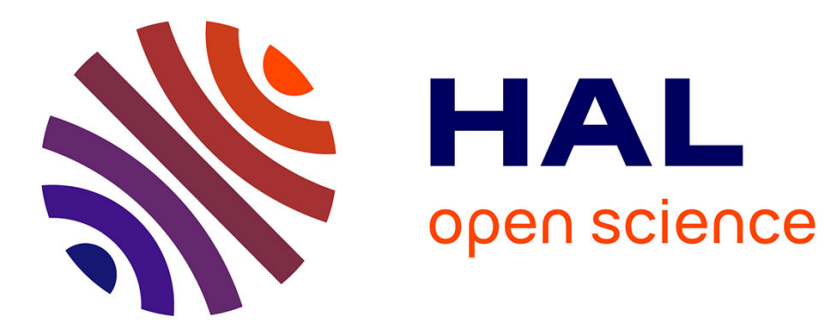

\title{
Un regard sur la trajectoire de l'informatique éducative au Brésil
}

\author{
Joana Peixoto
}

\section{To cite this version:}

Joana Peixoto. Un regard sur la trajectoire de l'informatique éducative au Brésil. STICEF (Sciences et Technologies de l'Information et de la Communication pour l'Éducation et la Formation), 2006, 13, 11 p. hal-00696412

\section{HAL Id: hal-00696412 https://hal.science/hal-00696412}

Submitted on 11 May 2012

HAL is a multi-disciplinary open access archive for the deposit and dissemination of scientific research documents, whether they are published or not. The documents may come from teaching and research institutions in France or abroad, or from public or private research centers.
L'archive ouverte pluridisciplinaire HAL, est destinée au dépôt et à la diffusion de documents scientifiques de niveau recherche, publiés ou non, émanant des établissements d'enseignement et de recherche français ou étrangers, des laboratoires publics ou privés. 


\title{
Un regard sur la trajectoire de l'informatique éducative au Brésil $\underline{1}$
}

\author{
Joana Peixoto LUniversité Catholique de Goiás, Brésil」
}

RÉSUMÉ : Cette rubrique présente l'analyse de l'évolution de l'informatique éducative (IE) au Brésil au travers des programmes officiels, des actions qui y sont menées et des théories qui les inspirent. Les projets en IE révèlent une sorte de vocation à résoudre la contradiction existante entre une position "nationaliste" et les réformes promues par l'état, après 64, qui elles, avaient une empreinte "internationaliste", plutôt sous influence Nord - Américaine.

MOTS CLÉS : Education et informatique, constructivisme pédagogique, innovation, enseignants formation.

ABSTRACT : This rubric presents the analysis of the evolution of education and informatics (EI) in Brasil through official programs, the theories that inspired them and the actions resulting from them. The projects in EI reveal a kind of vocation to solve contradictions between a "nacionalist" standpoint and the reforms promoted by the Brazilian State, after 64, which had an "internationalist" character, mainly under the influence of North America.

KEYWORDS : Education and informatics, pedagogical constructivism, innovation, Teacher's formation.

- La trajectoire de l'informatique éducative

- L'informatique éducative au Brésil : pistes pour l'analyse

- $\quad$ Références

- $\underline{\text { Sites Internet }}$

Au moment où nous observons la propagation des programmes de formation à distance au Brésil, le débat sur le recours aux technologies d'information et de communication (TIC) dans l'enseignement est plus que jamais marqué par son caractère communicationnel.

L'intérêt actuel se dirige donc, de façon toute particulière, vers la formation à distance, où la technologie est vue comme un outil permettant de palier à certaines difficultés. La formation à distance est notamment basée sur un discours pédagogique qui présente les vertus propres à ses dispositifs, "(...) en mettant en évidence leurs potentialités pédagogiques, comme le développement de l'autonomie des apprenants, par exemple ou l'augmentation de leur participation." (Pochon, p. 30, 2003)

Les recherches concernant la prise en compte des TIC dans les systèmes éducatifs de différents pays (Cros, 1997) (Cantin et al, 1999) (Guir, 2002) (Pouts - Lajus, 2002) (Baron et Bruillard, 2002) (Baron et Bruillard, 2003) (Charlier et Peraya, 2003) (Lagrange et Grugeon, 2003) (Pochon, 2003) (OCDE, 2004) sont plutôt prudentes : les possibilités d'une analyse comparative sont restreintes mais elles indiquent déjà que, malgré la configuration d'une tendance globale, les contextes sociaux sont assez diversifiés et les conditions politiques sont assez contrastantes pour générer une mise en œuvre particulière des projets de TIC en éducation. 
Comme les tentatives de généralisation sont considérées comme décevantes (Baron et Bruillard, 1996), "l'avenir de l'ordinateur dans l'éducation semble donc devoir passer d'avantage par des convergences et des actions locales que par la planification nationale." (Gabriel, p. 106, 1998)

Nous nous proposons ici d'analyser la trajectoire de l'informatique éducative au Brésil au travers des programmes officiels, des actions qui y sont menées et des théories qui les inspirent.

\section{La trajectoire de l'ínformatique éducative}

Au Brésil, les premières initiatives dans le domaine de l'Informatique Educative (IE), ont commencé dans les années 70. Puis, au cours des années 80, l'IE s'est développée au travers de diverses activités, lui permettant ainsi d'affirmer une identité propre.

Le Projet $\operatorname{Logo}^{\underline{2}}$ peut être considéré comme étant la première proposition formelle ayant comme objet d'étude : l'informatique dans l'éducation au Brésil. Ce projet marque une nette transformation des règles, qui jusqu'à ce moment prévalaient dans la construction du discours de l'informatique par rapport à son articulation avec l'éducation. Les pratiques discursives instituant l'informatique comme une technologie devant être maîtrisée et comme une connaissance devant être assimilée, se transforment par le surcroît des discours qui prônent l'informatique comme étant une technologie éducationnelle, c'est - à - dire comme étant une ressource de production d'apprentissage, comme un moyen de développement de l'intelligence des enfants.

Cette conception est fondée sur un discours qui a conquis des espaces significatifs dans le champ éducationnel. Il s'agit du constructivisme pédagogique, tributaire de la psychologie du développement de Piaget, lequel, dans les années 80 , a pu être considéré comme le principe de vérité sur le développement psychologique enfantin. Ce principe est présent dans l'éducation brésilienne et, depuis les années 90 , inspire une bonne partie des réformes de curriculum établies au Brésil et dans d'autres pays.

Il s'agit d'un mouvement discursif évoluant de l'apprentissage de l'informatique vers l'apprentissage au travers de l'informatique.

Le Logo, pris comme une philosophie éducationnelle, porte un discours suffisamment puissant pour influencer diverses expériences sur l'utilisation de l'ordinateur dans l'éducation brésilienne et contribuera, en ce sens, à influencer une bonne partie des projets d'IE au Brésil.

Conséquemment au besoin de formation des ressources humaines dans le secteur de l'informatique, le Secrétariat Spécial de l'Informatique (SEI) a créé en mars 1980, la Commission Spéciale no 1ㄹ, ayant comme finalité d'être un segment de soutien au ministère de l'éducation et de la culture (MEC) et au Secrétariat de SEI, avec comme objectif de "(...) recueillir des informations (...) et de générer les normes et les directives du nouveau et large champ qui s'ouvrait à l'éducation." (Brasil, p. 3, 1985)

Les recommandations exprimées dans le rapport de cette Commission indiquent que les activités liées à l'informatique dans l'éducation doivent être cadrées par des valeurs culturelles, socio - politiques et pédagogiques, propres à la réalité brésilienne. Derrière ces recommandations, nous retrouvons le discours qui met en valeur la potentialité du marché de l'informatique dans le pays, cherchant ainsi à rompre avec les liens de dépendance économique internationale.

En août 1981 est réalisé le Premier Séminaire National de l'Informatique dans l'Education, avec le soutien du Secrétariat Spécial de l'Informatique, du Ministère de l'Education et du Conseil National de la Recherche Scientifique et Technologique (CNPQ).

En 1984, le projet EDUCOM (Education et Ordinateurs) a mis en place des projets pilotes - Nucleus Interdisciplinaires - dirigés vers la recherche et la formation de ressources humaines dans des Universités ${ }^{4}$. Sur cette base, chaque université, tout en suivant les recommandations des Séminaires, 
développera des projets différents.

En 1986, le Programme d'Action Immédiate en Informatique et Education des $1^{\text {er }}$ et $2^{\text {ème }}$ degrés (PAIE) est créé, dirigé vers la formation des enseignants et vers la mise en place des infrastructures de support dans :

- les Secrétariats de l'Education des états - Centres d'Informatique Appliquée à l'Education des 1er et 2ème degrés (CIED) ;

- les écoles techniques fédérales - Centres d'Informatique Appliquée à l'Education dans l'Education Technologique (CIET) ;

- les universités - Centres d'Informatique Appliquée à l'Education dans l'Education Supérieure (CIES).

Après une revitalisation du financement, des ressources nouvelles ont été crées afin de soutenir: la recherche, la production de logiciels avec l'organisation d'un Concours National de Logiciel Educatif et la formation des enseignants par la mise en place du Projet FORMAR. Ce dernier a été mis en œuvre à travers deux cours de spécialisation en informatique et éducation, qui ont eu lieu à l'UNICAMP en 1987 et 1988, ayant pour but de former les professeurs des secrétariats d'état de l'éducation et ceux des écoles techniques. Les professeurs formés devaient par la suite prendre la responsabilité de structurations des Centres, où ils étaient chargés de définir leur programmes de formation, coordonner la recherche et la production de logiciels éducatifs.

En 1988, l'Organisation des Etats Américains (OEA) a invité le MEC à évaluer le projet d'Informatique Appliquée à l'Education Basique de Mexico. A partir de cette initiative, un projet multinational de coopération technique et financière a été formulé, intégré par huit pays américains, au cours des années 1990 à 1995.

En 1989, le MEC a édité le Programme National de l'Informatique dans l'Education - PRONINFE, ayant pour objectif :

- la promotion du développement de l'informatique éducative et son utilisation dans les systèmes publics de l'enseignement (1er, 2ème, 3ème degrés et éducation spéciale) ;

- l'émergence des infrastructures de support dans les écoles, soutenues par les Centres ;

- la formation continue et permanente des enseignants. 


\begin{tabular}{|c|c|}
\hline Premier Séminaire (1981) & ème Séminaire (1982) \\
\hline $\begin{array}{l}\text { l'indication que les activités de } \\
\text { l'informatique dans } \\
\text { l'éducation devraient être } \\
\text { balisées par des valeurs } \\
\text { culturelles, sociopolitiques et } \\
\text { pédagogiques de la réalité } \\
\text { brésilienne; } \\
\text { la nécessité de faire prévaloir } \\
\text { la question pédagogique en } \\
\text { dépit des questions techniques } \\
\text { dans la planification des } \\
\text { actions ; ren de } \\
\text { la reconnaissance } \\
\text { l'ordinateur comme un moyen } \\
\text { d'élargissement des fonctions } \\
\text { d'enseignant et jamais comme } \\
\text { une façon de s'y substituer. } \\
\text { les expériences dans ce } \\
\text { domaine devraient être } \\
\text { développées par } \\
\text { universités reconnues et } \\
\text { devraient avoir lieu dans des } \\
\text { écoles des ler et 2ème degrés. }\end{array}$ & $\begin{array}{l}\text { l'ordinateur doit être envisagé par } \\
\text { l'école comme une ressource } \\
\text { auxiliaire au processus éducatif et } \\
\text { jamais comme une finalité en elle } \\
\text { - même, c'est - à - dire que } \\
\text { l'ordinateur devrait être utilisé } \\
\text { afin de développer des habiletés } \\
\text { intellectuelles spécifiques, } \\
\text { requises par les différents } \\
\text { contenus curriculaires; } \\
\text { les applications ne devraient pas } \\
\text { concerner uniquement les écoles } \\
\text { des ler et 2er degrés, mais elles } \\
\text { devraient être étendues à tous les } \\
\text { degrés et à toutes les modalités } \\
\text { d'enseignement, avec } \\
\text { caractère interdisciplinaire. } \\
\text { la création de projets pilotes dans } \\
\text { les universités. }\end{array}$ \\
\hline
\end{tabular}

Tableau 1 : recommandations produites par les Séminaires Nationaux de l’Informatique dans l'Education

En 1990, le MEC a approuvé le 1er Plan d'Action Intégrée de l'Informatique et l'Education - PLANINFE - avec les mêmes principes du PRONINFE, afin de couvrir la période de 1991 à 1993. L'accent a été mis sur la formation des enseignants par le biais d'un programme intensif impliquant les universités, les secrétariats d'état à l'éducation, les écoles techniques, et les entreprises représentées par le Service National de l'Industrie (SENAI) et le Service National du Commerce (SENAC). En dépit de l'absence partielle de sources de financement et du manque d'expérience préalable, le PRONINFE peut être considéré comme une phase pilote qui aura duré plus d'une décennie et qui aura lancé les bases de la culture de l'informatique éducative au Brésil.

Le Programme National de Informatique Educative (PROINFO) a été lancé en avril 1997. Il avait pour objectif de distribuer cent mille ordinateurs aux écoles acceptant de se soumettre à un processus sélectif, en présentant une planification technologique liée à un projet pédagogique. Ce projet devait présenter leur investissement concernant la mise à disposition des installations physiques et la libération de l'emploi du temps des enseignants, cela afin qu'ils puissent participer au processus de formation. Les projets ont été sélectionnés par des commissions de jugement constituées par les états et homologués par le MEC.

Afin de pouvoir porter assistance à ces écoles, ainsi que pour leur suivi, le MEC a conçu des Nucleus de Technologie Educationnelle (NTE). Ces derniers fournissent aussi l'accès à l'internet.

La formation des ressources humaines devait se faire en deux étapes. Tout d'abord, les professeurs «multiplicateurs » ont été formés en informatique éducative. Ils faisent partie des équipes des NTE et ils devaient à leur tour former les «professeurs facilitateurs». Au cours d'une séconde étape des 
«facilitateurs » ont été désignés par les écoles, afin d'entraîner leurs collègues, mais aussi pour développer l'utilisation de l'informatique dans les salles de cours.

Selon Santos (Santos, 2003), le PROINFO est le Programme qui, actuellement, fait l'effort en IE le plus significatif dans le monde. Mais il est confronté aux mêmes difficultés rencontrées au cours d'autres expériences ayant eu lieu au Brésil ou dans d'autres pays. Par exemple, à São Gonçalo, une ville située dans l'état de Rio de Janeiro, des ordinateurs ont été livrés à des écoles n'ayant pas accès à l'énergie électrique (Pereira et Monteiro, 2004). Deux ans après la livraison des ordinateurs, ils n'étaient toujours pas installés. Dans d'autres écoles, le réseau électrique ne pouvait pas supporter l'installation de 10 ordinateurs simultanément. Les résultats, atteints par les actions gouvernementales, sont encore, en terme de quantitatif, très modestes, si l'on considère les dimensions continentales de ce pays ${ }^{\underline{5}}$.

\begin{tabular}{|c|c|}
\hline PRON & 96 - 2002) \\
\hline $\begin{array}{l}44 \text { Centres d'Informatique } \\
\text { dans l'éducation mis en place, } \\
\text { la plupart avec connexion à } \\
\text { l'Internet; } \\
400 \text { Sous centres mis en place, } \\
\text { dont un grand nombre, par des } \\
\text { gouvernements d'état et des } \\
\text { municipalités (Parmi eux, } 87 \\
\text { sont dans l'état de Rio Grande } \\
\text { do Sul); } \\
400 \quad \text { laboratoires } \\
\text { d'informatique éducative dans } \\
\text { des écoles publiques; } \\
\text { plus de } 10.000 \text { professionnels } \\
\text { préparés pour travailler avec } \\
\text { l'informatique éducative, y } \\
\text { compris des chercheurs ayant } \\
\text { un niveau de DEA ou de } \\
\text { doctorat. }\end{array}$ & $\begin{array}{l}2.200 \text { professeurs } \\
\text { multiplicateurs } \\
140.000 \text { professeurs dans les } \\
\text { écoles, dépassant l'objectif } \\
\text { prévu de } 25.000 . \\
9.000 \text { gestionnaires et } 10.000 \\
\text { techniciens de maintenance. } \\
4.641 \text { écoles ont été équipées } \\
\text { de laboratoires, dans } 1.116 \\
\text { villes. } \\
6 \text { millions d'élèves et } 215.000 \\
\text { professeurs en ont bénéficié ; } \\
350 \text { NTE ont été équipés, } \\
\text { dépassant les 250 prévus à } \\
\text { l'origine; } \\
3.515 \text { écoles du Proinfo sont } \\
\text { connectées à l'Internet. }\end{array}$ \\
\hline
\end{tabular}

Tableau 2 : résultats atteints par les deux derniers Programmes en Informatique et éducation

Concernant les actions internationales le Proinfo a proposé :

- RIVED (Réseau International Virtuel d'Education) - Projet multilatéral entre le Brésil, le Pérou et le Venezuela, ayant pour objectif de produire des solutions basées sur l'utilisation de la télématique pour l'enseignement des Sciences et des Mathématiques au niveau du secondaire.

- LTnet (Learning Technologies Network) - Environnement virtuel bilingue ayant pour objectif l'échange d'expériences et la formation d'éducateurs et de chercheurs. Projet bilatéral entre les Etats - Unis et le Brésil, devenu par la suite, une organisation non gouvernementale.

- Webeduc (Education sur le Web - projet bilatéral entre la France et le Brésil, ayant des finalités similaires au LTnet. Une des actions de ce projet a été l'ouverture de l'espace Langue Etrangère dans le programme brésilien TV Ecole, utilisant le cours de français basique Reflets, fourni par l'Ambassade de France. 
En outre, différents projets multilatéraux ont été financés par l'OEA. Enfin, nous pouvons observer que le Proinfo est un programme qui s'approprie le discours des chercheurs en IE au Brésil : celui qui soutient les pouvoirs transformateurs soit des technologies, soit de certains types de formations, comme l'atteste cet extrait du texte d'introduction du Rapport des Activités du Proinfo 1996 - 2002 :

«Le Proinfo ne se destine pas à réinventer la machine à enseigner, mais à faire en sorte que les professeurs réalisent mieux leur noble mission, en orientant les élèves pour qu'eux, appuyés par les nouvelles technologies de l'information et de la communication, deviennent de vrais citoyens, créatifs et indépendants, capables d'apprendre au cours de toute leur vie et de vivre dans une société dont le quotidien dépend, chaque fois plus, de la technologie. »

\section{L'ínformatique éducative au Brésil : pistes pour I'analyse}

La politique d'IE au Brésil a fécondé des contradictions. La politique brésilienne de l'IE avait une position «nationaliste », en opposition avec les réformes promues par l'Etat après 1964, qui, elles, avaient une empreinte internationaliste, plutôt sous influence Nord - américaine. C'est - à - dire que, l'Etat brésilien affiche l'intention de promouvoir une informatisation éducationnelle de direction nationale, à l'inverse de ce qu'il prétendait pratiquer dans l'enseignement général. Cette idéologie, portant un double discours, sert alors à nier les relations sociales du Brésil, qui sont plutôt marquées par sa dépendance envers le capital international.

Cette contradiction indique un jeu de forces existant entre l'Etat et la société civile. Le résultat est un discours faisant l'articulation entre les nouvelles technologies et les droits sociaux, le pluralisme idéologique et la diversité culturelle. Les projets en IE révèlent, alors, une sorte de vocation à résoudre cette contradiction, présente au sein des rapports sociaux.

L'exigence d'une pensée globale, alliée aux demandes d'actions locales, nous met au sein d'un mouvement contradictoire, qui coïncide avec le panorama socio - politique international. Nous ne pouvons plus nier l'attachement des méthodes et des techniques d'enseignement à une vision du monde ou à un projet politique de société. Nous ne pouvons pas non plus, ne pas reconnaître les liens entre l'actuelle dissémination sociale des TIC et le projet néolibéral. La pédagogie ne peut plus être ni techniciste ni naïvement nationaliste.

Almeida et Valente (Almeida et Valente, 1997) font l'analyse des facteurs présents dans les cours de formation d'enseignants proposés par les programmes officiels d'informatique et éducation du Brésil, mettant l'accent sur la forte influence des cours FORMAR I et FORMAR II sur la politique et sur les actions futures puisque les professionnels formés dans ces cours "(..) sont les responsables de la dissémination et de la formation des nouveaux professionnels dans le domaine (...)".

Nous sommes en accord avec eux, quand ils reconnaissent que ces cours ont présenté une vision large sur les différents aspects impliqués dans l'IE, autant du point de vue de l'ordinateur, que du point de vue pédagogique. Une telle caractéristique a permis aux élèves - eux mêmes, enseignants dans des lycées, des écoles techniques et des universités - de vraiment réfléchir à la possibilité de mettre en place des projets d'IE.

D’un autre côté, les cours étaient trop concentrés et ils ont été réalisés dans de grands centres urbains et universitaires, dans des locaux éloignés du lieu de travail des participants. Nous avons observé qu'une partie des départements d'origine des participants n'a pas mis en place de laboratoires ou l'a fait avec un grand décalage de temps, ce qui a généré de la méfiance et un manque de motivation pour développer un futur travail.

Les professeurs multiplicateurs arrivent avec enthousiasme à l'école et font de grands efforts pour 
transmettre leurs connaissances à leurs collègues ; mais ils n'arrivent pas à déclencher un processus où l'ensemble des professionnels des établissements d'enseignement s'implique. Les professeurs devant être formés ne dépassent pas l'état de passivité, et ainsi ne permettent pas d'établir une communication plus interactive et ouverte avec les multiplicateurs.

L'utilisation de l'ordinateur dans les collèges et lycées, quand il a lieu, a un caractère encore plus instrumental. Dans le cas où une méthodologie de projets de travail est mise en place, une bonne partie des projets est développée d'abord dans les salles de cours et, c'est uniquement après que les élèves aient maîtrisé les activités travaillées avec les professeurs, qu'ils utilisent les laboratoires pour reproduire sur l'ordinateur ces mêmes activités développées auparavant.

Il faut aussi insister sur le fait qu'en général, le laboratoire est présenté comme une place différenciée du reste de l'école; soit parce qu'il est doté d'équipements nouveaux, récemment livrés (entre autre, parce qu'ils ont été délivrés aux écoles avec presque deux ans de retard), soit en raison du mythe, encore présent, que le travail avec l'ordinateur demande une intelligence supérieure.

Tout cela contribue au fait que plusieurs professeurs n'utilisent pas le laboratoire d'informatique, parce que, d'une part ils ne savent pas l'utiliser et d'autre part ils allèguent de ne pas avoir le temps pour apprendre ou encore, résistent aux changements que son utilisation demanderait, et donc à une rupture dans leur routine de travail. La plupart des professeurs utilisent comme prétexte le manque de temps ou d'opportunité pour se consacrer à l'apprentissage de l'IE. Mais on peut aussi remarquer qu'il existe des professeurs ayant le désir de se tenir informés, de se moderniser et d'être disponibles pour se confronter avec les contraintes inhérentes aux changements.

Les conceptions sur l'IE présentes dans les directives établies par le PROINFO, sont en accord avec plusieurs critiques du système éducationnel, indiquant que l'école doit être à chaque fois plus familiarisée avec les innovations arrivant à tous les niveaux de la société, compte tenu du fait qu'il lui revient de préparer l'individu à agir de façon consciente et dynamique dans la société.

Un tel programme cherche à mettre en place de nouveaux processus éducationnels pour la préparation des professionnels de l'enseignement, tenant compte du fait que les TIC fournissent à l'école les bases pour un changement épistémologique; il s'agit de dépasser les relations d'enseignement et d'apprentissage contenus dans un paradigme de communication de masse, qui est essentiellement de transmettre l'information dans un processus d'interaction et de coproduction de la connaissance.

Pourtant, la réalité nous montre un autre scénario. On peut ainsi relever l'importance des politiques publiques de l'éducation et de leurs déploiements sociaux, considérant le ré-ordonnancement des bases productives, notamment du fait de l'internationalisation de l'économie et de l'incorporation des TIC par toutes les sphères de la société.

On peut aussi considérer les nouvelles bases qui caractérisent, aujourd'hui, le secteur productif: la nouvelle orientation économique dessinée, à partir de la globalisation de l'économie et des politiques néolibérales. Les technologies et, notamment, l'informatique conservent des convergences historiques avec le système d'éducation formelle et ont des présuppositions similaires.

L'école s'est fondée sur le rationalisme des Lumières et diffuse la croyance qu'elle même est une des conditions essentielles à la promotion de la démocratisation, permettant d'atteindre le statut de citoyen, sous la tutelle de l'Etat. Mais, nous observons des changements fondamentaux dans la vision de la scolarisation. Non plus la scolarisation proclamée par les idéaux égalitaires de l'ère des Lumières, mais une nouvelle scolarisation érigée à partir d'un nouveau réglage de l'établissement scolaire. Une scolarité qui propose une éducation plus syntonisée, plus adéquate et plus conforme aux changements des systèmes productifs.

Dans ce contexte, la production discursive autour de l'insertion d'un nouvel artefact culturel - l'ordinateur 
- dans l'éducation, lui attribue le sens d'un nouveau dispositif capable de déterminer et de modifier les expériences d'apprentissage. Le discours de l'IE émerge, donc, de cette conjonction entre un discours de nature économique, profondément syntonisé avec les changements des systèmes productifs et la représentation de l'apprenti, venant de la psychologie de développement piagétienne.

L'introduction de l'informatique dans l'éducation tend vers la modification des fondements pédagogiques. Devant l'expérience de la crise de la modernité et du surgissement de nouveaux modèles interprétatifs de la réalité, l'informatique est considérée, par certains auteurs, comme un nouveau langage, celui qui permettra de surmonter une telle problématique, en élaborant une nouvelle façon d'accéder à la connaissance.

Les objectifs attendus par le Secrétariat d'Enseignement à Distance (que coordonne le PROINFO) sont de faire arriver à l'école publique toute la contribution que les méthodes, les techniques et les technologies de l'éducation à distance peuvent fournir pour la construction d'un nouveau paradigme propre à l'éducation brésilienne. Les lignes d'action du Secrétariat d'Enseignement à Distance sont fondées sur l'existence d'un système technologique chaque fois moins cher, plus accessible, de maniement facile et capable ainsi d'apporter à l'école un important potentiel didactico - pédagogique; d'élargir les opportunités là où il y a de faibles ressources; de familiariser le citoyen avec la technologie existante dans son quotidien; de donner des réponses flexibles et personnalisées à des personnes qui exigent une plus grande diversité de types d'éducation, de types d'information et d'entraînement ; d'offrir les moyens d'actualiser rapidement la connaissance; d'étendre les espaces éducationnels; de motiver les professionnels et les élèves à apprendre continuellement et ce, quelle que soit l'étape de leur vie.

Cette préoccupation n'est pas présente uniquement dans le secteur de l'éducation à distance. Dans le système de l'éducation nationale, la formation revient aussi comme une priorité. L'enseignement est pensé comme une habilitation rapide pour ceux qui ont besoin de pénétrer très vite sur le marché du travail. Ces propositions, indissociables de l'utilisation des TIC, sont simplifiées en substituant aux cours conventionnels la formation sur le lieu de travail.

Par rapport à la formation des enseignants, cette politique peut être traduite par le privilège d'une formation plus légère et moins chère, comme la "formation en service, à distance et au travers de cours plus rapides" (Pretto, 1999). Cette politique de formation valorise plus l'entraînement, que la formation stricto sensu. Elle est soutenue par les recherches de la Banque Mondiale, pour lesquelles la performance des élèves dépend moins de la formation des professeurs que des matériaux pédagogiques utilisés. D'où l'investissement en masse sur des matériaux didactico - pédagogiques: depuis les livres, jusqu'à l'ensemble des ressources multimédias.

Dans le discours de la Banque Mondiale, l'essor de l'éducation - une exigence pour la concession de prêts aux pays demandeurs - passe par l'utilisation de technologies plus efficaces, pensées comme une manière de casser le monopole du professeur dans la transmission de la connaissance. C'est à dire, la mise un œuvre d'un modèle qui donne plus d'importance aux moyens d'enseigner qu'aux médiations pédagogiques.

Or, il n'y a pas de neutralité dans la technique. Les technologies et les langages ont de multiples dimensions qui doivent être appropriées de façons différentes. Selon le modèle en question, la complexification des matériaux d'enseignement s'articule avec un allégement de la formation. Ainsi, les médiations pédagogiques deviennent vides et les multimédias sont utilisés comme des illustrations, pour la transmission rapide des connaissances.

S'il est vrai que l'école doit s'investir dans la formation par différents chemins et langages, et plus encore dans l'élargissement de ses conceptions de langages, dans la mise en place d'une médiation possible grâce aux technologies numériques, cela implique un approfondissement et non un allègement de la formation professionnelle. 
Les ressources numériques arrivent dans les écoles où la formation des enseignants a lieu, mais il n'y a pas de mobilisation des connaissances et des compétences multiples des acteurs impliqués. En cela, il est difficile d'espérer que des changements essentiels arrivent concernant l'organisation et la pédagogie des écoles, notamment vers la constitution de processus d'apprentissage plus ouverts et interactifs.

Les temps et les espaces de l'école restent presque les mêmes, et la technologie demeure comme un appendice. Dès lors, il est possible d'expliquer l'existence de professeurs ouverts aux changements mais pourtant pessimistes par rapport à la possibilité de changements dans l'école, soit en raison de l'absence d'une infrastructure physique requise pour la mise en œuvre de l'IE, soit par des difficultés d'ordre politique, bureaucratique et pédagogique auxquelles ils pourraient être confrontés, dans la mesure où ils acceptent le défi d'introduire les TIC dans leur pratique éducative.

\section{Références Bibliographie}

ALMEIDA, Fernando José et VALENTE, José Armando (1997). Visão Analítica da Informática na Educação no Brasil : a questão da formação do professor. Revista Brasileira de Informática na Educação, n. 1. http://gmc.ucpel.tche.br/rbie-artigos/nr1-1997/valente.htm (consulté en mai 2002)

BARON, Georges - Louis et BRUILLARD, Eric (1996). L'informatique et ses usagers dans l'éducation. Paris, PUF, 1996.

BARON Georges - Louis, dir., BRUILLARD Eric (2002). Les technologies en éducation, perspectives de recherche et questions vives : actes du symposium international francophone. Paris, INRP.

BARON, Georges - Louis et BRUILLARD, Eric (2003). Les technologies de l'information et de la communication en éducation aux USA : éléments d'analyse sur la diffusion d'innovations technologiques. Revue Française de Pédagogie, n. 146, p.37-49.

BRASIL. Ministério da Educação (MEC) / FUNTEVE. Un Relato do Estado Atual da Informática no Ensino no Brasil. Brasília, DF, 1985.

CANTIN, Judith et al. (1999). Les TIC en pédagogie universitaire et en formation à la profession enseignante au Québec : mythes et réalités. Revue AC - TICE, $\mathrm{n}^{\circ}$ 8. http://www.ac-nancy-metz.fr/AcTice/ (consulté en novembre 2004)

CAVALCANTE, Meire \& GUIMARÃES, Arthur. Manutenção de computadores do Proinfo expira em 2004. [en ligne] avril, 2003. http://novaescola.abril.com.br/index.htm?noticias/abr_03_4/index_1. (consulté en novembre 2004)

CHARLIER, Bernadette \& PERAYA, Daniel (éds.) (2003). Technologie et innovation pédagogique. Dispositifs innovants de formation pour l'enseignement supérieur. Bruxelles, De Boeck Université, 2003.

CROS, Françoise. L'innovation en éducation et formation (1997). Revue Française de Pédagogie, n. 118, p.127-156.

CYSNEIROS, Paulo Gisleno (2003). Programa Nacional de Informática na Educação: novas tecnologias, velhas estruturas. In BARRETO, Raquel Goulart. Tecnologias educacionais e educação à distância : avaliando políticas e práticas. 2e éd. Rio de Janeiro, Editora Quartet.

GABRIEL, Philippe (1998). Enseignement et informatique pour tous : où en sommes - nous ? Revue Française de Pédagogie, n. 124, p.99-108.

GUIR, Roger (2002). Pratiquer les TICE. Former les enseignants et les formateurs à des nouveaux usages. Bruxelles, De Boeck Université, 2002. 
LAGRANGE, Jean - Baptiste et GRUGEON, Brigitte (2003). Vers une prise en compte de la complexité de l'usage des TIC dans l'enseignement. Une métha - analyse des publications d'innovation et de recherche en mathématiques. Revue Française de Pédagogie, n. 143, p.101-111.

OCDE (2004). Organisation de coopération et de développement économiques. L'OCDE constate que l'utilisation des TIC est « décevante » dans les établissements d'enseignement secondaire du second degré. http://www.oecd.org/document/18/0,2340,fr_2649_201185_26304850_1_1_1_1,00.html (consulté en mars 2004)

PEREIRA et MONTEIRO. Atitudes de professores e alunos do ensino fundamental face à introdução da informática na sala de aula. http://www.nce.ufrj.br/ginape/publicacoes/trabalhos/

CesarMaterial/relatos\%20Coinfe\%5CRelatos\%20do\%20IV-Coinfe.doc (consulté en novembre 2004)

POCHON, Luc - Olivier (2003). Quelques repères historiques et culturels concernant les NTIC et leur usage dans l'éducation et la formation. Cahiers de Psychologie, Neuchâtel, Université de Neuchâtel, n. 39. http://www.unime.ch/psy (consulté en décembre 2004)

POUTS - LAJUS, Serge (2002). Les yeux plus grands que le ventre. Les TICE dans le dispositif scolaire. Education Permanente, n. 152, p.85-94.

PRETTO, Nelson (1999). Educação e inovação tecnológica : um olhar sobre as políticas públicas brasileiras. Revista Brasileira de Educação, n. 11, p. 75-85. http://www.ufba.br/ pretto/textos/rbe11.htm (consulté en mai 2002)

SANTOS, Gilberto Lacerda (2003). A internet na escola fundamental : sondagem de modos de uso por professores. Educ. Pesqui., vol. 29, n. 2, p.303-312.

VALENTE, José Armando (org.) (2003). Formação de educadores para utilização da informática na escola. Campinas, Nied - UNICAMP/ OEA.

\section{Sites Internet}

Les articles concernant l'IE au Brésil

http://www.nied.unicamp.br/publicacoes/

http://portal.mec.gov.br/seed/index.php?option=content \&task=view\&id=136\&Itemid=273

http://www.edutec.net/

http://lsm.dei.uc.pt/ribie/pt/textos/textos.asp

Le site officiel du Ministère de l'éducation

http://www.mec.gov.br/seed/tvescola/

Le site du Programme National d'Informatique Educative

http://www.proinfo.gov.br 
1 Cet article fait partie des études menées dans le contexte de la thèse «Les formateurs parlent. Les représentations des formateurs d'enseignants dans le domaine de l'informatique appliquée à l'éducation » , dirigée par Geneviève Delaunay-Jacquinot et soutenu par la Capes (Coordenação de Aperfeicoamento de Pessaoal de Nivel Superior), du Ministère de l'Éducation au Brésil. Il a fait l'objet d'une relecture de Georges-Louis Baron.

$\stackrel{2}{2}$ Le Projet Logo a été développé par l'Université de Campinas au cours des années 1973 à 1982. Son objectif initial a été d'introduire le langage Logo et de l'adapter à la réalité brésilienne. Cela a commencé par une étude restreinte avec des enfants et par la suite avec des professeurs qui ont été formés à la production de matériel didactique, selon la philosophie Logo.

$\underline{3}$ Cette Commission, présidée par la SEI, avait, comme membres, des représentants des universités suivantes: Université de São Paulo, Université Catholique de Rio de Janeiro, Université Fédérale de Minas Gerais, Université Fédérale de Pernanbuco, Université Fédérale du Rio Grande do Sul, Faculté Technologique.

${ }^{4}$ Universités : Fédérale de Rio Grande do Sul (UFRGS), Fédérale de Rio de Janeiro (UFRJ), Fédérale de Pernambuco (UFPE), Fédérale de Minas Gerais (UFMG) et de l'Etat de Campinas (UNICAMP).

5 Selon Alberto Castilho Siqueira, coordinateur général de l'accompagnement et de l'évaluation de l'éducation à distance du Ministère de l'Education, "Il existe 180.000 écoles publiques au Brésil dont seulement 4.638 ont bénéficié du Proinfo jusqu’à 2002." (Cavalcante et Guimarães, 2003)

\section{- A propos de l'auteur}

Joana Peixoto est enseignante à L'Université Catholique de Goiás-Brésil. Elle a soutenu sa thèse début 2005 à Paris 8. Depuis, elle travaille avec la discipline "Èducation, communication et médias" dans les cours de formation d'enseignants et elle s'est chargé de l'élaboration du projet du Centre d'Éducation aux médias du cours de Pédagogie de la même université.

Adresse : Universidade Católica de Goiás - Departamento de Educação. Avenida Universitária, n. 1069, CP 86, Setor Universitário. Goiânia - Goiás - Brésil. 74605-010.

Courriel : joanagyn@yahoo.com.br

Toile : http://www.mec .gov.br/seed/tvescola/

\section{Référence de l'article :}

Joana Peixoto, Un regard sur la trajectoire de l'informatique éducative au Brésil, Revue STICEF, Volume 13, 2006, ISSN : 1764-7223, mis en ligne le 07/05/2006, http://sticef.org

(C) Revue Sciences et Technologies de l'Information et de la Communication pour l'Éducation et la Formation, 2006 